\title{
Dense clutter multi-target tracking algorithm based on improved fuzzy clustering data association
}

\author{
Xuchao Kanga , Guangjun He \\ Air and Missile Defence College, Air Force Engineering University, Xi’an, China \\ a841451491@qq.com
}

Keywords: fuzzy clustering; multi-target tracking; data association; kalman filter

\begin{abstract}
In order to improve the accuracy and rapidity of multi-target tracking in clutter environment, the fuzzy clustering algorithm is used to obtain the relative degrees of different measurement target memberships, and the fuzzy correlation probabilities of measurement values and targets are calculated in combination with public measurement influence factors. Then using fuzzy association information combined with Kalman filtering, each target is separately tracked through the weighted fusion method to achieve the state update of the target. The simulation results show that the combination of fuzzy clustering and joint probability data improves the fastness of the target clustering in the relatively dense environment of clutter, and the accuracy of the target tracking is ensured by the combination of Kalman filter and the weighting of the influence factor.
\end{abstract}

\section{Introduction}

Multi-target tracking in dense clutter environment has always been a problem in the target tracking field. For example, in the ultra-low altitude environment, the target tracking will be affected by ground and sea clutter. The signals captured by the radar include the real measurement. False measurement caused by clutter makes it difficult to accurately track the target. Many scholars have done a lot of research on the distinction between false goals and goals, but they all have problems of large computational complexity and complex target differentiation, which is not conducive to real-time tracking of targets. Probabilistic Data Association Algorithm (PDA), which is proposed to track targets in a clutter environment. The algorithm is computationally intensive, but it is easy to follow the wrong target in a clutter-intensive ultra-low altitude environment. Based on the PDA, the joint probability data association algorithm (JPDA) can solve the target tracking in the clutter-intensive environment. However, as the number of targets increases, the JPDA algorithm is too computationally intensive, which is not conducive to real-time tracking of targets. Hard to apply. Thus, the application of fuzzy clustering to data association (FDA) has emerged, greatly reducing the complexity of multi-target tracking calculations.

Literature (2) obtains the membership degree between each measurement point and the target prediction position by fuzzy clustering, and uses it as a weight coefficient to weight the information quantity of the effective echo to achieve the update of the state estimation of the target. However, in the clutter-intensive ultra-low altitude environment, clutter has a great influence on the accuracy of state estimation. Literature (4) proposes a method based on the combination of fuzzy clustering and Kalman filtering based on the size of information entropy. Literature (3) considers the influence of public measurement on the target estimation. Based on the fuzzy clustering, the scale factor is added, and multi-level clustering is performed according to the position information of the target group and the motion direction information. However, most of the literature does not consider the difference in the weight of the common measurement for the target estimation of the multi-target in the short-range and long-distance situations, which seriously affects the accuracy of the tracking. In this paper, a method of combining fuzzy clustering association with Kalman filtering is proposed for the ground (sea) clutter-intensive ultra-low altitude environment. By analyzing the multiobjectives in the two cases where the distance is relatively close, the public measurement pair The 
influence of the target estimation introduces the influence factor, which further improves the accuracy of multi-target tracking. Using the fuzzy clustering algorithm, the target can be quickly classified and the real-time tracking can be guaranteed.

\section{Problem description}

Assume that in the multi-target tracking system, there are $C$ tracking targets, and the motion state equation of the target $i(1,2,3 \cdots M)$ at time $k$ is

$$
X_{k+1}^{i}=F_{k}^{i} X_{k}^{i}+G_{k}^{i} W_{k}^{i}
$$

Where $X_{k}^{i}$ is the state vector of the target $i$ at the time $k, F_{k}^{i}$ is the state transition matrix of the target $i$ at the time $k, W_{k}^{i}$ is a Gaussian white noise that is independent of each other and has a mean of zero, and the covariance matrix is $Q(k)$, and $G_{k}^{i}$ represents the system noise drive matrix. Suppose the observation equation of the target $i$ at time $k$ is

$$
Z_{k}^{i}=H_{k}^{i} X_{k}^{i}+V_{k}^{i}
$$

$Z_{k}^{i}$ is the measurement vector of the target $i$ at time $k, H_{k}^{i}$ is the measurement matrix of the target $i$ at time $k, V_{k}^{i}$ is the observed noise matrix, and is also the Gaussian white noise with a mean of zero, and the covariance matrix is $R(k)$.

The problem solved in this paper is to measure $n$ measurement $Z_{k}(j)(j=1,2 \ldots \ldots n)$ at time $k$.The observation includes dense clutter (false target). Under the premise that the clutter cannot be distinguished from the real target measurement, the idea of fuzzy clustering is used. The observations (including clutter) are clustered, and the membership degree of each observation relative to the target is obtained as the fuzzy association probability. The influence of the public measurement on the target in the two cases of distance and near distance is analyzed, and the influence factor is used to reconstruct the fuzzy association probability. The matrix is then combined with the Kalman filter method to weight the state estimation of different observations to obtain the estimation state of the target at the next moment. The target trajectory is estimated separately to realize the real-time tracking of the ultra-low altitude multi-target.

\section{Improved fuzzy clustering association algorithm}

\subsection{Fuzzy C-means clustering}

Fuzzy C-means clustering (FCM) is the most widely used clustering method in fuzzy clustering. The core idea is to use the similarity between one or several features of many samples to divide the target into target prediction. The location-centric class completes the classification to implement data association. Given a sample set $Z=\left(z_{1}, z_{2}, z_{3} \ldots z_{n}\right)$, the cluster center is $C_{i}$, the number of clusters is $C$, and the membership of the data $Z(j)$ for the i-th cluster center is $\mu_{i n}$, and satisfies:

$$
\begin{array}{r}
\mu_{i j} \in[0,1](1 \leq i \leq c, 1 \leq j \leq n) \sum_{j=1}^{c} \mu_{i j}=1(1 \leq j \leq n) \\
0 \leq \sum_{j=1}^{n} \mu_{i j} \leq n(1 \leq i \leq c)
\end{array}
$$

Fuzzy partition matrix is $U=\left(\mu_{i j}\right)_{c ? n}$, defining fuzzy functions

$$
J(u, v)=\sum_{i=1}^{c} \sum_{j=1}^{n} u_{i j}^{m} d_{i j}{ }^{2}
$$


$d_{i j}$ is the Euclidean distance between sample $j$ and target $i, v_{i}$ is the clustering center of the target $i$, and $m$ is the fuzzy index, which satisfies $1 \leq m \leq \infty$. The fuzzy classification is completed by minimizing the objective function. The specific process is as follows:

(1) Determine the target $C$, the state prediction value at the following moment as the initial clustering center, and select the appropriate fuzzy index m. From the cluster validity test, the best value range of $m$ is $\left[\begin{array}{ll}1.5 & 2.5\end{array}\right]$, usually take $m=2$, give The iteration stops the threshold $\varepsilon$.

(2) Initialize the membership matrix $U$, calculate the distance of each data to the cluster center $d_{i j}$, and calculate the membership matrix, $t=0$.

(3) Update the membership function matrix $U=\left(\mu_{i j}\right)_{c ? n}$

$$
\mu_{i j}=1 / \sum_{k=1}^{c}\left[\frac{\left\|z_{j}-v_{i}\right\|}{\left\|z_{j}-v_{k}\right\|}\right]^{2 /(m-1)}
$$

(4) Update cluster center

$$
v_{i}=\sum_{j}^{n} \mu_{i j}^{m} z_{j} / \sum_{j=1}^{n} \mu_{i j}^{m}
$$

(5) If $\left|v_{i}(t)-v_{i}(t+1)\right|<\varepsilon$, the iteration ends, the fuzzy partition matrix is obtained, otherwise go to step 3.

In this paper, under the premise that the number of targets (the number of clusters) is known, the predicted position of the target at the next moment is the cluster center, and the Euclidean distance of each measurement and cluster center is used as a measure to measure the membership of the target degree

\subsection{Improved fuzzy clustering association algorithm}

Establish a target elliptical tracking gate to confirm the effective observation distance $r_{i}$ of each target and $\left\|r_{i}\right\| \leq \tau_{i}$ reduce the wild value. Where is the observed threshold for the target.

Establishing a measurement attribution matrix $\Lambda$

$$
\begin{gathered}
\Lambda=\left[\begin{array}{llll}
\vartheta_{11} & \vartheta_{12} & \ldots & \vartheta_{1 n} \\
\vartheta_{21} & \vartheta_{22} & \cdots & \vartheta_{2 n} \\
\cdots & \ldots & \cdots & \cdots \\
\vartheta_{M 1} & \vartheta_{M 2} & \cdots & \vartheta_{M n}
\end{array}\right] \\
\vartheta_{i j}= \begin{cases}1, & \mathrm{~d}_{\mathrm{ij}} \leq \mathrm{r}_{\mathrm{i}} \\
0 & \mathrm{~d}_{\mathrm{ij}}>\mathrm{r}_{\mathrm{i}}\end{cases}
\end{gathered}
$$

$\vartheta_{11}=1$ indicates that the observation gate $z_{1}$ falls into the tracking gate of the target $1, \vartheta_{23}=0$ that the observation $z_{3}$ does not fall into the tracking gate of the target 2 .

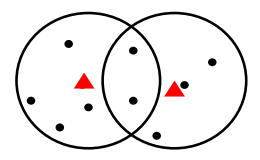

Figure 1 Distribution of measured values in the target confirmation area

In Figure 1, $\Delta$ represents the predicted position of the target, and the tracking gate is established centering on the target predicted position. • represents the measuring point at a certain moment (including clutter and real target measurement). Suppose that at a certain moment, the target has only one true measurement. The rest is a spurious measurement caused by clutter, and the measurement of the overlap area of the tracking gate is a common measurement.

Most of the traditional algorithms do not consider the coincidence of multi-target tracking gates, 
that is, the existence of public measurement. Although some scholars have considered the influence of public measurement on target estimation in the presence of public measurement, it does not consider the change of the weight of the common measurement on the target estimation when the multi-target is closer and farther, and affects the accuracy of tracking. . The improved fuzzy clustering association algorithm analyzes the difference of the influence weights of the public measurement on the target estimation under the multi-objective distance, and adjusts the weight of the public measurement in real time.

For two distant targets, it is considered that the probability of the public measurement area is small, which is usually considered as clutter. At this time, the scale factor is introduced to reduce the weight of the public measurement for the target estimation. However, when the two targets are close together, especially when crossing, the probability of non-public measurement being clutter is greater. At this time, the scale factor should be introduced to reduce the weight of non-public measurement.

When the target distance is far away, the measurement distribution is shown in Figure 2.

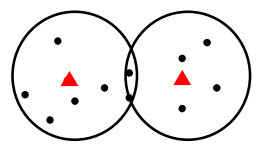

Figure 2 Measurement distribution when the targets are far apart

At this time, the deviation between the public measurement and the target prediction is large, and the possibility of clutter is high. The influence factor is introduced to reduce the weight of the public measurement.

When the target distance is close, the measured distribution is shown in Figure 3.

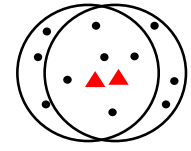

Figure 3 Measurement distribution when the target is close

At this time, the deviation between the non-public measurement and the target prediction is large, and the possibility of clutter is high. The influence factor is introduced to reduce the weight of the non-public measurement.

In order to represent the two scenarios of multiple targets at a distance, let $\varpi=\sum_{j=1}^{n} \vartheta_{a j}-\vartheta_{b j}$ (a, b is an integer from 1 to $M$ ), $\varpi$ indicates the number of non-public measures. According to the relationship between the number of non-public measures and the total number of measurements, it can be divided into the following two cases:

When $\varpi \geq \frac{n}{2}$, the number of target public measures is less than half of the total number of measurements, the targets are considered to be far apart, and the common measurement set

$$
\text { pub_set }=\left\{z(j) \mid \sum_{i=1}^{M} \vartheta_{i j}>1, j=1,2, \ldots n\right\}(8) \text { Introducing the influence factor } \varsigma_{\text {pub }} \text { to reduce the }
$$

weight of the public measurement

$$
\begin{gathered}
\varsigma_{p u b}=\left(1-\frac{\varpi}{n}\right) \\
\vartheta_{i j}^{*}=\varsigma_{p u b} \vartheta_{i j}
\end{gathered}
$$

When $\varpi<\frac{n}{2}$, the number of target public measures is greater than half of the total number of measurements, the targets are considered to be close together. Non-public measurement set 


$$
\text { nonpub_set }=\left\{z(j) \mid \sum_{i=1}^{M} \vartheta_{i j}=1, j=1,2, \ldots n\right\}(10
$$

Introducing impact factors $\varsigma_{\text {non-pub }}$ reduces the weight of non-public measures

$$
\begin{array}{r}
\varsigma_{\text {non-pub }}=\frac{\varpi}{n} \\
\vartheta_{i j}^{*}=\varsigma_{\text {non-pub }} \vartheta_{i j}
\end{array}
$$

Measurement distribution matrix after introducing influence factors

$$
\Lambda^{*}=\left[\begin{array}{cccc}
\vartheta_{11}{ }^{*} & \vartheta_{12}{ }^{*} & \ldots & \vartheta_{1 n}{ }^{*} \\
\vartheta_{21}{ }^{*} & \vartheta_{22}{ }^{*} & \ldots & \vartheta_{2 n}{ }^{*} \\
\cdots & \cdots & \ldots & \cdots \\
\vartheta_{M 1}{ }^{*} & \vartheta_{M 2}{ }^{*} & \cdots & \vartheta_{M n}{ }^{*}
\end{array}\right]
$$

Establish a measurement weight matrix

$$
\Pi=\left[\begin{array}{cccc}
\omega_{11} & \omega_{12} & \cdots & \omega_{1 n} \\
\omega_{21} & \omega_{22} & \cdots & \omega_{2 n} \\
\cdots & \cdots & \cdots & \cdots \\
\omega_{M 1} & \omega_{M 2} & \cdots & \omega_{M n}
\end{array}\right]
$$

$$
\omega_{i j}=\vartheta_{i j}{ }^{*} \mu_{i j}
$$

\section{Multi-target tracking algorithm based on fuzzy clustering}

Using FCM algorithm to obtain the measurement weight matrix, combined with Kalman filter, the joint correlation information is used to track the target separately for the uncertainty of measurement under clutter environment. The specific process is as follows:

Step1 Initialize each target state, given initial value $\hat{X}^{i}(0), P^{i}(0),(i=1,2, \ldots M)$

Step2 Predict the target state at the next moment as the clustering center for the next moment

$$
\hat{X}^{i}(k / k-1)=F^{i}(k / k-1) \hat{X}^{i}(k-1 / k-1)
$$

Step3 Observation prediction

$$
\hat{Z}^{i}(k)=H^{i}(k) \hat{X}^{i}(k / k-1)
$$

Step4 Determine the effective observation of each target, the maximum observation range $r_{i}$, and establish a measurement attribution matrix $\Lambda$.

Step5 Using fuzzy clustering to calculate the membership relationship matrix $U$ of each measure $\mathrm{z}(\mathrm{j})$ belonging to the target $i . U=\left(\mu_{i j}\right)_{c ? n}$

Step6 According to the relationship between the public quantity measurement and the total measurement, a scale factor $\varsigma_{\text {pub }} \varsigma_{\text {non-pub }}$ is introduced to establish a measurement weight matrix $\Pi$.

Step7 One-step prediction of mean square error

$$
\begin{aligned}
P^{i}(k / k-1)= & F^{i}(k / k-1) P^{i}(k-1) F^{i}(k / k-1)^{T} \\
& +Q^{i}(k-1)
\end{aligned}
$$

Step8 Calculate the filter gain

$$
\begin{aligned}
K^{i}(k)= & P^{i}(k / k-1) H^{i}(k)^{T}\left(H^{i}(k) P^{i}(k / k-1) H^{i}(k)^{T}\right. \\
& \left.+R^{i}(k)\right)^{-1}
\end{aligned}
$$


Step9 Using different weights of relative targets for weighted fusion, the state update value of the $\mathrm{i}$-th target is obtained.

$$
\hat{X}^{i}(k)=\hat{X}^{i}(k / k-1)+K^{i}(k) \sum_{j=1}^{M} \omega_{i j}(k)\left[Z_{j}(k)-\hat{Z}^{i}(k / k-1)\right]
$$

Step10 Estimated mean square error

$$
\begin{aligned}
& P^{i}(k)=\left[I-K^{i}(k) H^{i}(k)\right] P^{i}(k / k-1)+K^{i}(k)\left[\sum _ { j = 1 } ^ { M } \omega _ { i j } ( k ) \left[Z_{j}(k)\right.\right. \\
& \left.-\hat{Z}^{i}(k / k-1)\right]\left[Z_{j}(k)-\hat{Z}^{i}(k / k-1)\right]^{T}-\left[\sum_{j=1}^{M} \omega_{i j}(k)\right. \\
& \left.\left.g\left[Z_{j}(k)-\hat{Z}^{i}(k / k-1)\right]\right]\left[\sum_{j=1}^{M} \omega_{i j}(k)\left[Z_{j}(k)-\hat{Z}^{i}(k / k-1)\right]\right]^{T}\right] K^{i}(k)^{T}
\end{aligned}
$$

\section{Simulation results and analysis}

In order to verify the reliability of the algorithm, two scenarios are set:

Scenario 1: Verifying the reliability of the algorithm when the two targets are far apart (no intersection).

Suppose there are two targets with uniform linear motion. The initial positions of target 1 and target 2 are $(1500,4000),(1000,900)$, the target 1 horizontal velocity is $300 \mathrm{~m} / \mathrm{s}$, and the vertical velocity is $200 \mathrm{~m} / \mathrm{s}$, target 2 horizontal velocity is $200 \mathrm{~m} / \mathrm{s}$, vertical velocity is $100 \mathrm{~m} / \mathrm{s}$, sampling period is $0.2 \mathrm{~s}$, membership degree matrix $U$ index is set to 2 , maximum iteration number is set to 100 , iteration termination condition membership degree The minimum change is 1e-5.

In the case where the clutter is sparse, when the clutter coefficient $\lambda=2$ is taken, the target tracking trajectory and error curve are as follows:
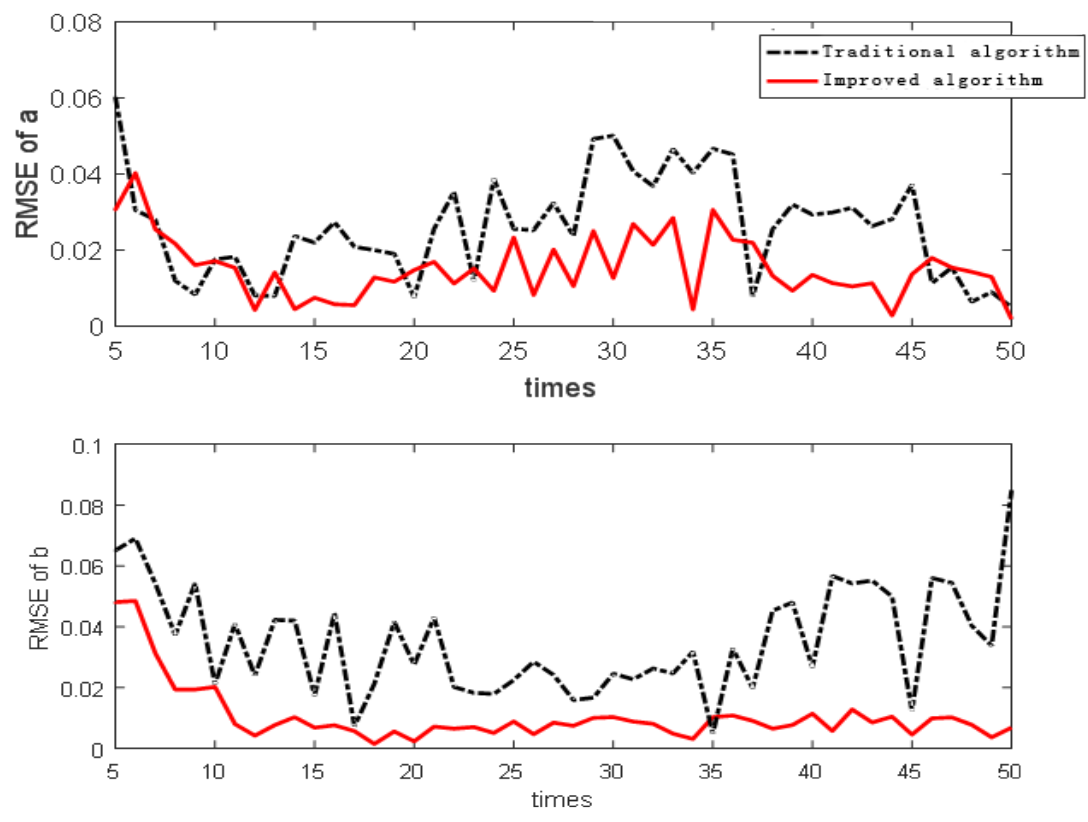

Figure 4 Error curves of targets

In the case of dense clutter, when the clutter coefficient $\lambda=5$ is taken, the target tracking trajectory and error curve are as follows: 

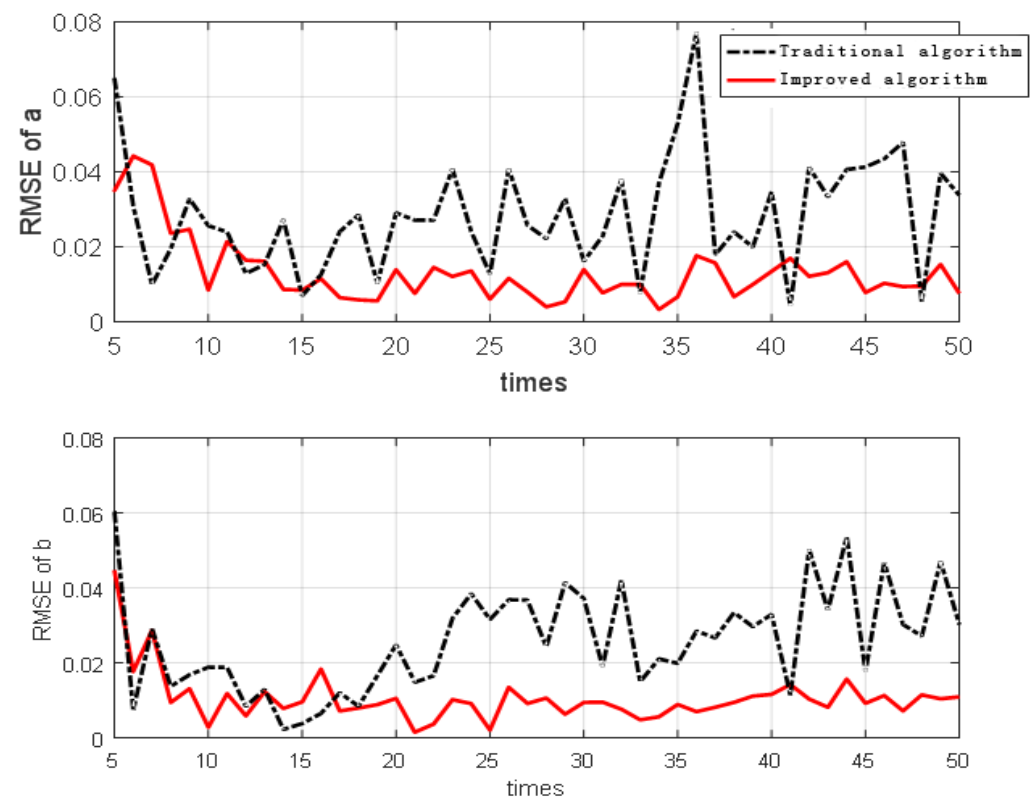

Figure 5 Target error curve in dense clutter environment

Scenario 2: Verifying the reliability of the algorithm when the two targets are close together (there are intersections)

In the case where the clutter is sparse, when the clutter coefficient $\lambda=2$ is taken, the target error curve is as follows
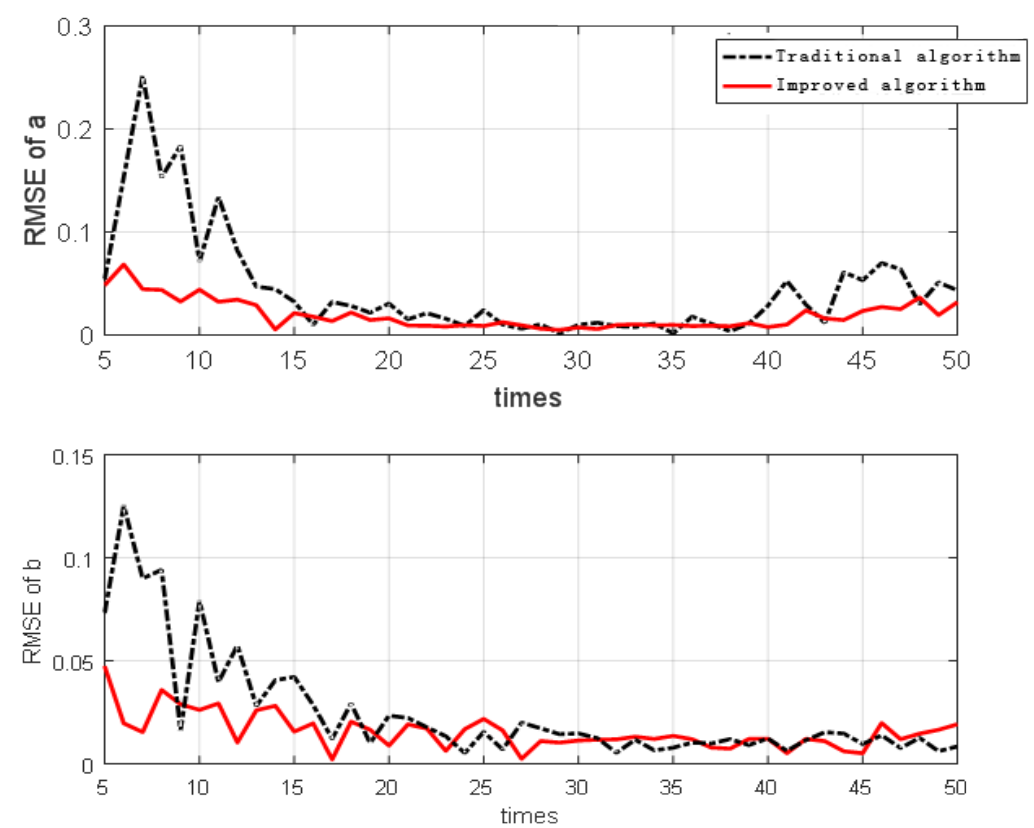

Figure 6 Error curves of targets

In the case of dense clutter, when taking $\lambda=5$, the target error curve is as follows: 

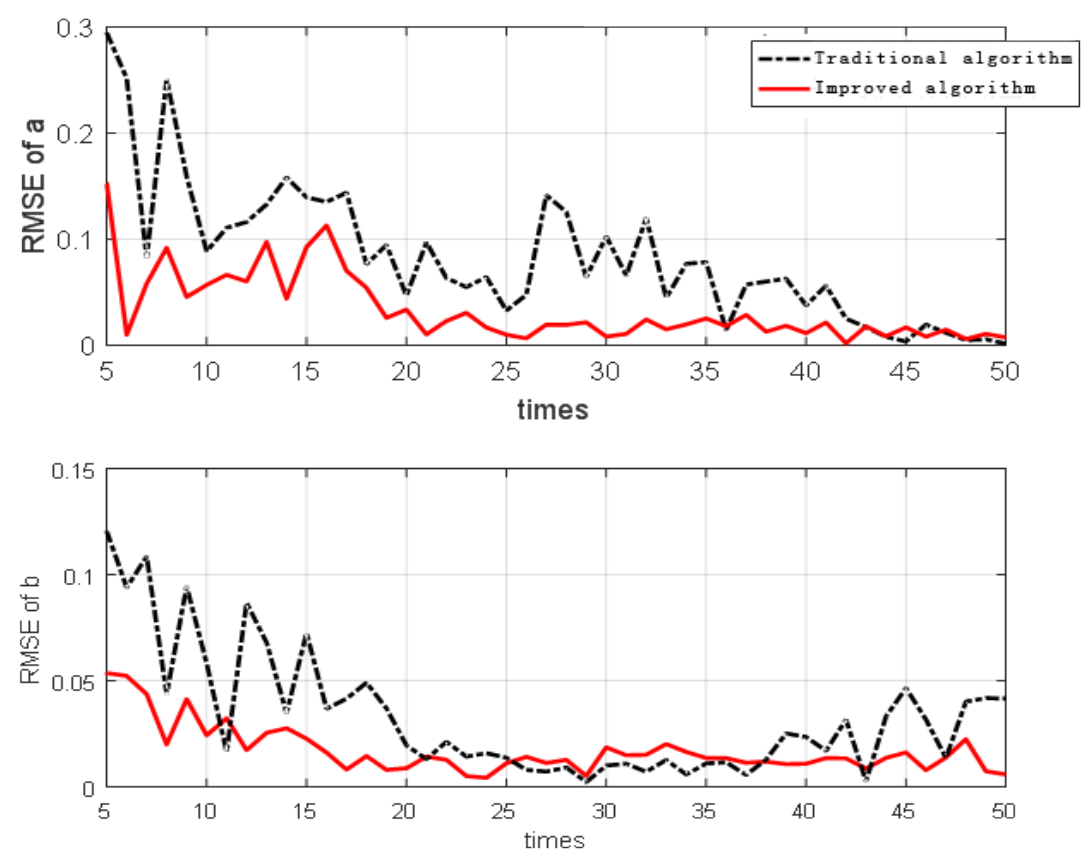

Figure 7 Target error curve in dense clutter environment

Through the simulation analysis of the two scenarios, the improved algorithm can reflect the target's motion trajectory more accurately than the traditional multi-target tracking algorithm without considering the common influence factor, whether it is for close-range or long-distance targets. And it has better tracking effect for multi-target tracking under clutter intensive, which proves that the proposed tracking algorithm is more suitable for tracking multi-targets in dense clutter interference environment.

\section{Conclusion}

This paper focuses on the multi-target tracking problem in the case of dense clutter, uses fuzzy clustering to obtain the target association probability, introduces the influence factor near the different distances to reconstruct the fuzzy association probability matrix, and uses the reconstructed fuzzy association probability as the weight. Kalman filter is used to estimate the state of different measurements at a certain time as the target's estimation state, to achieve the state update of the target, and the fuzzy clustering method is used to realize the real-time tracking. The correlation probability obtained by introducing the influence factor is used as the weight. The state estimation of different observations is weighted to ensure the accuracy of the target tracking.

\section{References}

[1] A. Rodriguez and A. Laio, "Clustering by fast search and find of density peaks", Science, Vol.344, No.6191, pp.1492-1496, 2014.

[2] Zhang Jungen, Ji Hongbing. Passive multi-target tracking based on independent particle filtering and fuzzy clustering[J].Journal of XIDIAN University, 2010,37(4):636-641.

[3] Liu JUN, Liu Yu ,et al.Joint Probablilistic Data Association Algorithm Based on All-neighbor Fuzzy clustering in Clutter[J]. Journal of Electronics \& Information Technology, 2016,38(6):14381445.

[4] Chen Xiao, Li Yaan,et al. A Fast Multi-Target Tracking Algorithm Based on Maximum Entropy Fuzzy Clustering[J]. Journal of Northwestern Polytechnical University, 2017,35(4):629-634.

[5] Mahler R. PHD Filters of Higher Order in Target Number[J]. IEEE Trans on Aerospace and 
Electronic Systems,2007,43(4):1523-1543

[6] Qian Pengjiang,Jiang Yizhang,Deng Zhaohong,et al.Cluster Prototypes and Fuzzy Memberships Jointly Leveraged Cross-Domain Maximum Entropy Clustering[J]. IEEE Trans on Cybernetics, 2016, 46(1):181- 193

[7] Mcgee J A,Luginbuhl T E,Dibiase J H,et al.A Modified PDAF Algorithm for Measurement Covariances that Vary within aTime Scan[J].IEEE Trans on Aerospace and Electronic Systems, 2012, 48(1) : 906- 912

[8] Son H S,Park J B,Joo Y H. Tracking Algorithm Compensating Acceleration for 3D Maneuvering Target with PSO-FCM[C]//2012 IEEE International Conference on Fuzzy Systems, Brisbane, Australia,2012: 1-7

[9] Habtemariam B,Tharmarasa R,Thayaparan T,Mallick M,Kirubarajan T. A Multiple-Detection Joint Probabilistic Data Associ-ation Filter[J]. IEEE Journal of Selected Topics in Signal Processing,2013,7(3): 461-471 